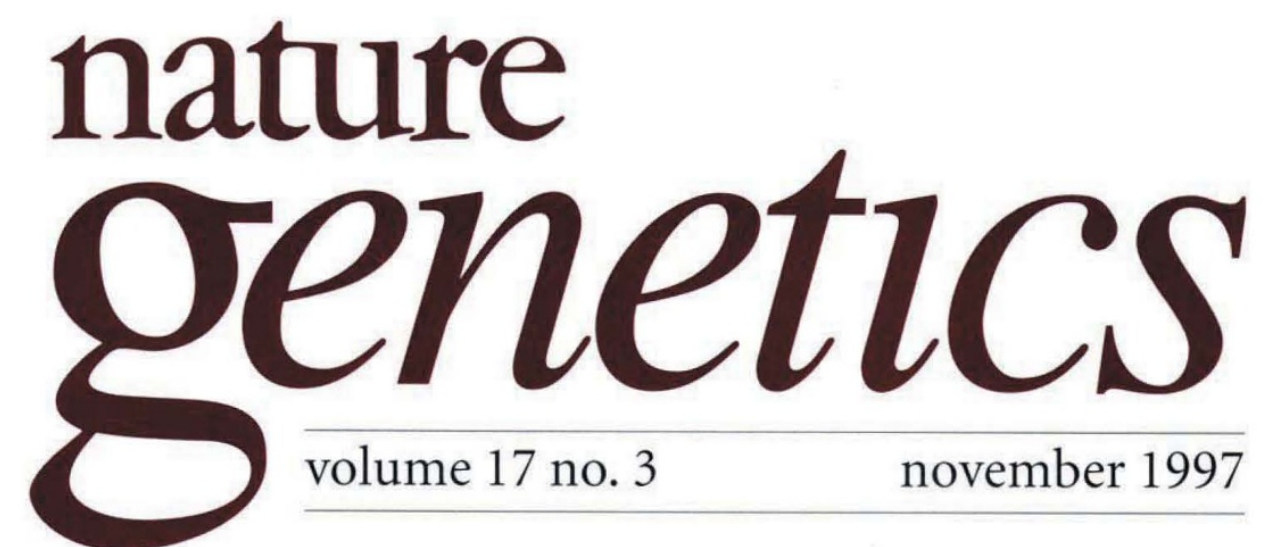

\title{
No stranger to controversy
}

Those working on $B R C A 1$ and $B R C A 2$, the genes most frequently mutated in hereditary breast cancer and associated with 3-8\% of all breast cancers, have become accustomed to the contention that follows every 'breakthrough' in the field ${ }^{1}$. With this historical backdrop in their wake, participants in the First International Workshop on the Function of BRCA1 and BRCA2, descended upon the tranquil fens of Cambridge (UK) and prepared for the challenge.

One controversy that has embroiled the field for the past couple of years continues unabated -is BRCA1 located in the nucleus or the cytoplasm? The odds are now in favour of nuclear localization, a conclusion supported by Cindy Wilson (UCLA School of Medicine), who described a comprehensive characterization of every antiBRCA1 antibody she "could get [her] hands on". It would appear that

First International Workshop on the Function of BRCA1 and BRCA2, Cambridge, UK, September 11-12, 1997; sponsored by the Breakthrough Breast Cancer charity.

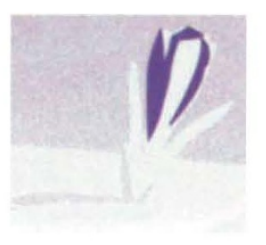
previous discrepancies in BRCA1 localization data may have arisen from differences in antibody specificity, variable immunodetection techniques, the low concentration of the endogenous protein and artefacts generated from overexpressing the protein in cells. For those unconvinced, there will have been a chance to explore this issue further at a BRCA1 antibody workshop, sponsored by the National Cancer Institute, in October as this article goes to press. From studies with their anti-BRCA2 antibodies, Inder Verma (Salk Institute) and Alan Ashworth (Cancer Research Campaign Centre, Sutton) presented evidence at the Cambridge meeting that BRCA2 is also localized to the nuclear compartment.

The new fascination in the field is dots, dots and more dotsnuclear foci, to be precise. BRCA1 appears, together with RAD51 and BARD1, as discrete nuclear dots during $S$ phase of the cell cycle (see insert overpage). Ralph Scully (Harvard Medical School) described how, in response to DNA damage, the BRCA1/RAD51/BARD1 'dots' disperse and the proteins later reconvene on replicating $\mathrm{DNA}^{2}$. The precise function of BRCAl at these replicating DNA structures in damaged cells is unknown, although its co-localization with RAD51, a protein involved in recombination and repair, suggests that BRCA1 has a role in the repair of genetic damage. BRCA2, like BRCA1, also appears to be involved in DNA repair; Ashworth showed that cell lines derived from mice homozygous for a hypomorphic mutation in Brca2 are unable to combat the genomic damage caused by irradiation.

The inventory of proteins involved in the repair taskforce associated with BRCAl and BRCA2 is far from complete. According to Wen-Hwa Lee (University of Texas Health Science Center), BRCAl does not directly bind to RAD51, but does to Rad50. BRCA2, on the other hand, interacts with RAD51 and not Rad50. One possible scenario is that BRCAI binds BRCA2 binds RAD51; alternatively, BRCA1 binds Rad50 binds RAD51; or perhaps an unidentified 'middle man' may be involved. This theoretical matchmaking is, however, premature until the full complement of proteins associated with BRCA1 and BRCA2 is unmasked. Furthermore, BRCA1 is reputed to be a transcriptional activator and is known to liaise with the massive holoenzyme transcription complex ${ }^{3}$. Verma supported BRCAl's role as an activator of gene expression with evidence that BRCA1 binds p300/CBP and together they enhance transactivation. Thus BRCAl may not actually become involved 
with the hands-on molecular mechanics of DNA repair but may, rather, act as a foreman, activating the expression of genes responsible for reconstructing the damaged genome.

The balance appears to be tipped in favour of BRCA1 and BRCA2 functioning as 'caretakers' responsible for maintaining the integrity of the genome. There has, however, been a recent resurgence of BRCA1's earlier fame as a potential regulator of the cell cycle. Jonathan Licht (Mount Sinai Medical Center, New York) described BRCAl's ability to inhibit cell proliferation, when transfected into cells, by directly inducing the expression of $C D K N 1 A$ (also known as $p 21$ ), suggesting that it can bypass $\mathrm{p} 53$ to exert its own negative check-point control over cell growth ${ }^{4}$. While the exact relationship between BRCA1 and p53 in their control of the cell cycle remains ambiguous, Tim Crook (Institute of Cancer Research, Sutton) presented evidence that p53 mutations are frequently associated with familial $B R C A 1$-associated tumours, suggesting that perhaps the influence of BRCA1 and p53, although unlikely to be synergistic, may be additive and that the loss of both genes may be integral to the progression of tumorigenesis in inherited breast cancer.

It came as somewhat of a surprise to researchers busily dissecting out the function of BRCA1 in the laboratory to find out that it is already being tested in the treatment of cancer patients. Jeffrey Holt (Vanderbilt Cancer Center) described Phase-I clinical trials for ovarian cancer using recombinant retroviruses expressing BRCA1. Twelve patients with late-stage ovarian cancer received daily injections of large volumes of the retroviruses into their peritoneal cavities. Holt reported that subsequent tumour biopsies revealed evidence of gene transfer into the tumour and expression of the BRCAl protein. Preliminary evaluation of the patients suggests "a fair amount of disease stabilization", but the Phase-I study was primarily aimed at evaluating toxicity; Phase-II clinical trials are now under way to determine efficacy. One curious feature of this study was its use of a BRCA1 variant lacking a RING finger domain considered critical to BRCA1 function. Given that mutations in this region are associated with malignancy, it is paradoxical that the BRCAl variant may have a therapeutic effect in the treatment of cancer.

The heated exchange that followed Holt's presentation questioned the use of BRCA1 as a therapeutic tool when there is only rudimentary knowledge of its cellular role. There was also concern about the rationale of applying a DNA repair protein to try to alter the growth properties of mutated cells. In defence of the trials, Holt referred to studies conducted in mice, before the clinical trials, that demonstrated that the BRCA1-expressing retroviral vectors have anti-tumour activity ${ }^{5}$. Furthermore, BRCAl inhibits growth when overexpressed in cells, but Robert Weinberg (Whitehead Institute) pointed out that many proteins, if overexpressed ectopically, will perturb the growth of cells. Tak Mak (Ontario Cancer Institute) cautioned, from an immunologist's perspective, on the interpretation of the clinical data given that the introduction of a pathogen or large amounts of protein can induce cytokine production and, as a consequence, alter the growth of tumour cells.

There has been remarkable progress in the functional characterization of BRCA1 since its initial cloning in 1994 (ref. 6), but there is general disquiet among researchers in the field over the use of BRCA1 in clinical trials when there is only a preliminary understanding of its function. It could be argued that there are many medical treatments in use for which we have little knowledge of the precise mechanism by which they work. Not one successful gene therapy protocol, however, has made its way into routine medical practice, despite the enrolment of more than 2,100 participants in various gene therapy trials worldwide since 1990 (ref. 7). Certainly, a spectrum of factors influence the outcome of clinical trials. Nevertheless, as we progress towards the application of ever more sophisticated technology for treating cancer and genetic diseases, it is crucial that there be a scientific conceptual basis underlying the likelihood of success of a therapeutic rationale.

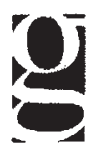

